\title{
Técnica Flexible de Zhang para el cálculo de parámetros intrínsecos y extrínsecos en el ajuste de dietas en cultivos de Oreochromis $S p$
}

\author{
Zhang's flexible technique for the calculation of intrinsic and \\ extrinsic parameters in the adjustment of diets in crops of \\ Oreochromis Sp
}

Recibido: 15 Ago 2017 - Revisado: 30 Sep 2017

Aceptado: 30 Oct 2017 - Publicado: 30 Dic 2017

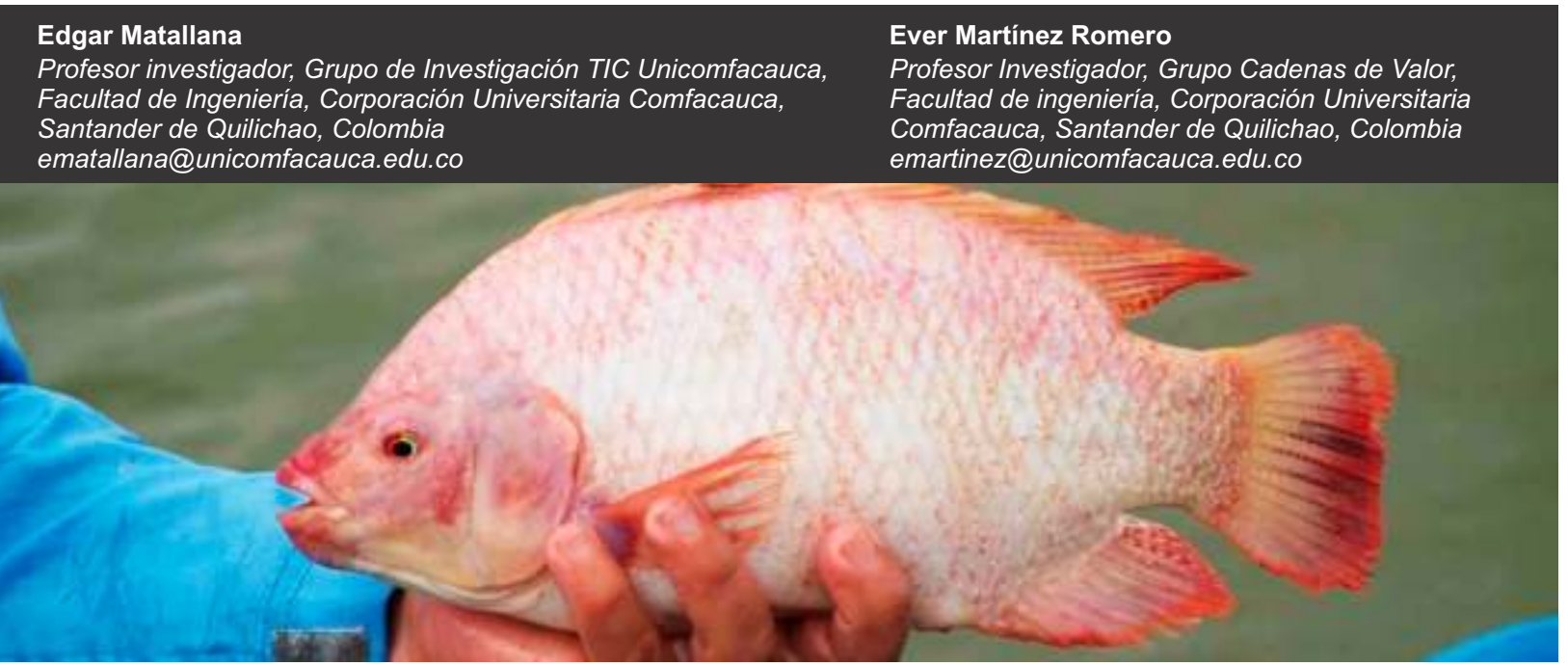

Resumen: El cálculo de los parámetros intrínsecos y extrínsecos de cámaras digitales usadas en los cultivos de Tilapias, facilita el ajuste de dichas cámaras a las medidas biométricas reales que se esperan obtener del cultivo. Con estos cálculos se busca crear un modelo matemático que permita relacionar las medidas en pixeles del sensor óptico de las cámaras con las medidas biométricas de las tilapias. Para encontrar la talla de la tilapia roja por medio de reconocimiento de imágenes digitales se determinaron las características internas, geométricas y ópticas de la cámara (Parámetros intrínsecos) y la posición de referencia de la cámara, con respecto al acuario o estanque (parámetros extrínsecos). El propósito era hallar un modelo matemático que permitiera obtener la información biométrica de las tilapias a partir de las imágenes digitales obtenidas por la cámara. Para este caso, se aplicó el método de calibración de Zhang, utilizando imágenes de un plano con patrones de intensidad específicos, capturados por la cámara en diferentes posiciones y orientaciones. Con los parámetros encontrados se buscó el modelo matemático que relacionara las medidas reales biométricas de las tilapias con el análisis de las imágenes digitalizadas.

Palabras claves: calibración de cámaras; visión por computador; Matlab toolbox.

Abstract: The calculation of the intrinsic and extrinsic parameters of digital cameras used in the cultures of Tilapias, facilitate the calibration of the cameras to obtain the real biometric measurements of the fish. The mathematical model allows to relate the measurements in pixels of the optical sensor of the cameras with the biometric measurements of the tilapia. To find the size of the red tilapia by means of recognition of digital images, the internal characteristics were determined, corresponding to the geometry and optics of the camera (intrinsic parameters). Likewise, the external characteristics of the camera were determined, corresponding to the reference position with respect to the aquarium or pond (extrinsic parameters). The objective was to find a mathematical model that would allow obtaining the biometric information of the tilapia from the digital images obtained by the camera. For this case, Zhang's calibration method was applied, using images of a plane with specific patterns, captured by the camera in different positions and orientations. With the parameters found, a mathematical model was developed to relate the real biometric measurements of the tilapia with the digitized images of the tilapia.

Key words: camera calibration; computer vision; Matlab toolbox. 


\section{INTRODUCCIÓN}

El Reconocimiento de Imágenes digitales es ampliamente utilizado en la industria y en la agroindustria para hacer conteos, como el de huevos en la industria avícola, para la selección de frutas identificación y selección de frutas dañadas, como en las cadenas frutícolas o para analizar la morfo-colorimetría de variedades de arroz, como en la industria arrocera.

El semillero de investigación ImasDmasl de la corporación Universitaria Comfacauca, en el departamento del Cauca - Colombia, está desarrollando un "Sistema para determinar por medio de reconocimiento de imágenes el ajuste de dietas en cultivos de tilapia roja -oreochromis $s p$-, el cual incluye el diseño de un algoritmo matemático para capturar imágenes por medio de cámaras digitales.

En el trabajo se muestra cómo se obtuvieron los parámetros intrínsecos y extrínsecos de la cámara Logitech c270, utilizando la técnica desarrollada por A. Zhang (2000) en A Flexible New Technique for Camera Calibration.

Los parámetros intrínsecos y extrínsecos de la cámara son utilizados para el desarrollo de un modelo matemático que permite calcular las medidas biométricas de las tilapias rojas, por medio de las imágenes digitales tomadas con la cámara Logitech c270.

\section{ANTECEDENTES}

\subsection{Parámetros Intrínsecos y Extrínsecos}

Los parámetros intrínsecos describen la geometría y óptica del conjunto cámara y sensor de adquisición de imágenes de la cámara Logitech C270, por otro lado, los parámetros extrínsecos dan la orientación y posición de la cámara donde ella estará ubicada para la toma de las imágenes de la tilapia roja. (Torres et al. 2002; Vega 2008; Cyganek y Siebert 2011.)

\subsection{Modelo Pinhole}

Un modelo muy sencillo pero práctico para representar una cámara es el conocido Modelo Pinhole, representado en la figura 1 , que relaciona la imagen de un objeto tridimensional en el mundo real $(\mathrm{Xm}, \mathrm{Ym}, \mathrm{Zm})$, la posición de la cámara $(\mathrm{Xc}$, $Y c, Z c)$, la del sensor (Xs, Ys), la del centro óptico (Cx, Cy) y de la imagen tomada (Xi, Yi).

De acuerdo con Tsai $R$ (1987), uno de los inconvenientes del modelo es que no se tienen en cuenta las características tecnológicas de las cámaras en el mercado, como, por ejemplo, el zoom óptico. Para el caso de este proyecto, el origen del sistema de coordenadas y el escalado o zoom para cada imagen son fijos en cada toma, por lo que el modelo matemático resultante se podrá considerar muy cercano a lo real.

Los parámetros intrínsecos agrupados en la matriz de calibración propuesta para el modelo de cámara Logitech C270 incluyen la información de los siguientes parámetros.

4 Ubicación del punto principal (Xm, Ym, Zm)

Escalado o zoom

D Distorsión óptica

(1) Distancia focal

Los parámetros extrínsecos se agrupan en la matriz de rotación y el vector de traslación que relacionan las coordenadas tridimensionales del objeto $(\mathrm{Xm} \quad \mathrm{Ym} \quad \mathrm{Zm})$ con las coordenadas tridimensionales de la cámara (Xc Yc Zc). (Gardel 200; Vega 2008)

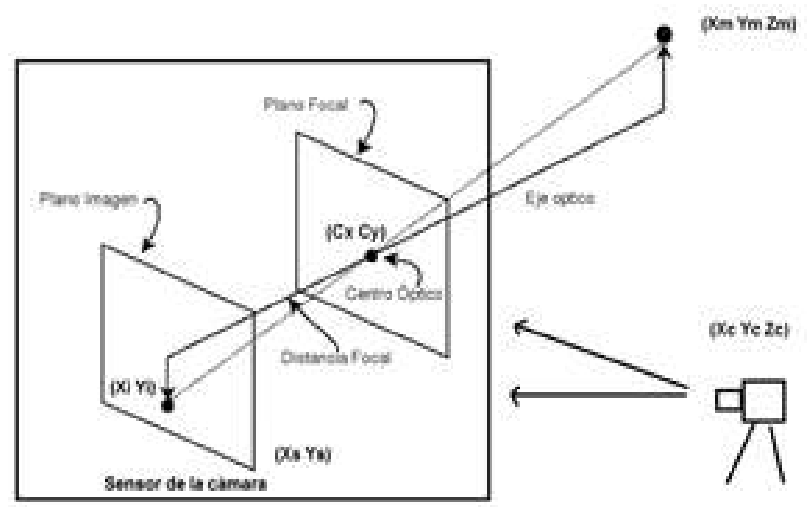

Fig 1. Representación Modelo Pinhole.

\subsection{Modelo Matemático}

Cualquier objeto tridimensional (Xm Ym Zm) fotografiado por la cámara en el mundo real puede representarse en el sistema de coordenadas de la cámara (Xc Yc Zc), por el producto de una matriz de rotación $R$ más la suma con un vector de translación $T$ : donde las matrices $R$ y $T$ son conocidas como parámetros extrínsecos, tal y como lo refleja la expresión 1.

$$
\left[\begin{array}{l}
X_{c} \\
Y_{c} \\
Z_{c}
\end{array}\right]=R\left[\begin{array}{l}
X_{m} \\
Y_{m} \\
Z_{m}^{m}
\end{array}\right]+T=\left[\begin{array}{lll}
r_{1} & r_{2} & r_{3} \\
r_{4} & r_{5} & r_{6} \\
r_{7} & r_{8} & r_{9}
\end{array}\right]\left[\begin{array}{l}
X_{m} \\
Y_{m} \\
Z_{m}
\end{array}\right]+\left[\begin{array}{l}
T_{x} \\
T_{y} \\
T_{z}
\end{array}\right]
$$

Si no se tienen en cuenta la distorsión radial y tangencial de los lentes de la cámara, las coordenadas ( $\mathrm{Xi}$ Yi) de la imagen en el sensor de la cámara se pueden encontrar con la siguiente expresión 2. 


$$
\left[\begin{array}{l}
x_{i} \\
y_{i} \\
1
\end{array}\right]=k\left[\begin{array}{ccc}
f_{c x} & 0 & C_{x} \\
r_{4} & f_{c y} & c_{y} \\
0 & 0 & 1
\end{array}\right]\left[\begin{array}{l}
X_{c} \\
Y_{c} \\
Z_{c}
\end{array}\right]
$$

Siendo fcx y fcy las distancias focales de la cámara, que incluye también el valor en $\mathrm{mm}$ del área del sensor óptico, $\mathrm{k}$ es un factor de escala zoom de la cámara-, Cx y Cy son los valores de las coordenadas del centro óptico de la cámara. Los anteriores valores son denominados parámetros intrínsecos de la cámara.

\section{Metodología}

\subsection{Ubicación}

La investigación se llevó a cabo en la ecoaldea Dar Amor - FUNDAMOR, ubicada en la vereda Mandiva, perteneciente al municipio de Santander de Quilichao.

La ecoaldea tiene un área de 10 hectáreas y en ella se realizan actividades agrícolas y pecuarias como cultivo de tomate, frijol, cacao, mango, cultivos gallinas ponedoras, cultivo de peces. Posee ecosistema de bosque con un área de tres hectáreas y posee tres afloramientos de agua.

\subsection{Descripción Área de Estudio}

El estanque tiene como área $12 \mathrm{~m}^{2}$, está construido en cemento y posee una entrada y una salida para el abastecimiento de agua y desagüe. Se sembraron 200 peces para una densidad de siembra de 16.6 peces $/ \mathrm{m}^{2}$

Se trabajó con dos cámaras cuya referencia es Logiteth c270, adaptadas para ser introducidas a los acuarios del cultivo

Las cámaras se instalaron a un PC marca DELL, vía puerto USB o IEEE 1394, para la adquisición y digitalización de las imágenes. La digitalización de la imagen se realizó por medio de las herramientas desarrolladas para esta función y que vienen con el módulo de visión de la National Instruments, este módulo contiene las librerías de $\mathrm{NI}$-IMAQ que funciona con la versión de LabVIEW 2012 o Matlab. Esta digitalización se realizó diariamente durante toda la etapa del proyecto.

\subsection{Cálculo de los Parámetros Intrínsecos y Extrínsecos}

Para el cálculo de los parámetros intrínsecos y extrínsecos de cada cámara Logitech C270 se utilizó el método flexible propuesto por Zhang (2000), que incluye los siguientes pasos:

(a) Imprimir un patrón geométrico definido y unirlo a una superficie plana. (b) Tomar una serie de imágenes del patrón geométrico desde diferentes orientaciones moviendo para ello la cámara o el patrón geométrico.

(c) Detectar los puntos importantes de la imagen tomada por la cámara.

(d) Estimar los parámetros intrínsecos y los parámetros extrínsecos.

A continuación, en la figura 2 , se muestra el patrón geométrico utilizado, que consiste en dibujos de cuadriculas de $25 \mathrm{~mm}$ de lado.

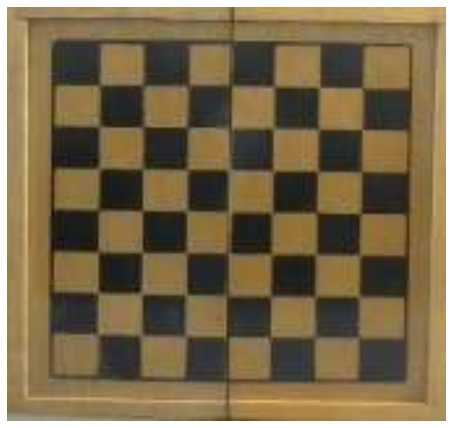

Fig 2. Imagen del patrón geométrico utilizado.

Para el cálculo de los parámetros extrínsecos el enfoque de la cámara se colocó a una distancia de $45 \mathrm{~cm}$ con respecto al sensor óptico de la misma. Se tomaron fotos de diferentes longitudes, a una distancia de alrededor de 45 centímetros, para encontrar la proporción entre la longitud real del objeto con la longitud captada en pixeles por el sensor óptico de la cámara. Estos valores se llevaron a una hoja de cálculo donde se encontró la función matemática que relaciona longitudes reales con valores de pixeles en el sensor óptico.

\section{Resultados}

Calibración de los parámetros intrínsecos de la cámara Logitech c270 utilizando la herramienta "Camera calibration toolbox for Matlab"

Para encontrar la talla de la tilapia roja por medio de reconocimiento de imágenes digitales se utilizó la cámara Logitech c270. Se determinaron las características internas, geométricas y ópticas de la cámara -parámetros intrínsecos- y la posición de referencia con respecto al acuario o estanque de la cámara -parámetros extrínsecos-. El objetivo era encontrar y resolver un modelo matemático que permita obtener la información biométrica de las tilapias, a partir de las imágenes digitales obtenidas por la cámara Logitech c270. El anterior procedimiento se conoce con el nombre de calibración de la cámara. En el caso del proyecto la técnica que se aplicó es conocida como el método 
de calibración de Zhang (Zhang 2000). Para esto, se utilizaron imágenes de un plano que tiene adherido un patrón en intensidad específico cuadros blancos y negros-), capturadas por la
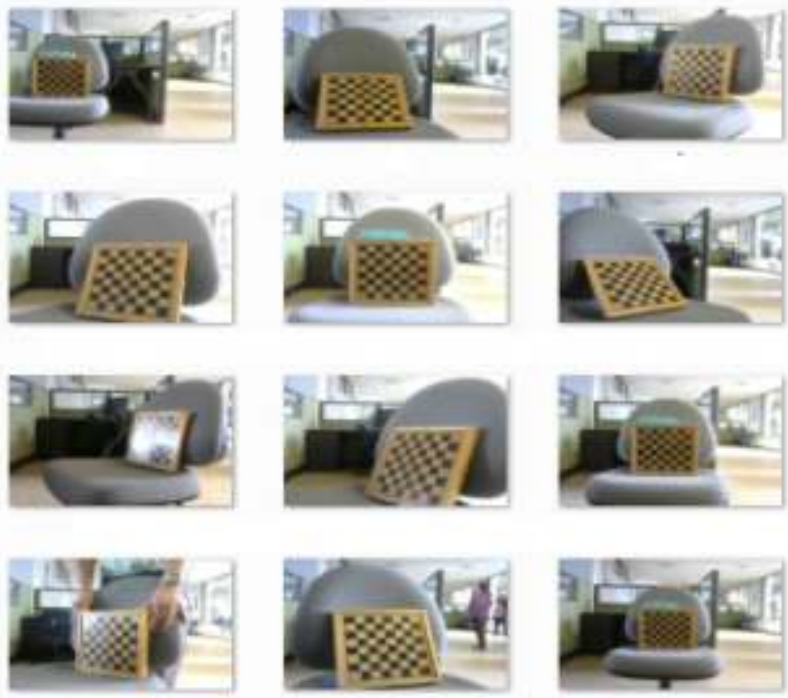

cámara en diferentes posiciones y orientaciones, el cual se muestra en la siguiente imagen. En la figura 3 se pueden observar diferentes posiciones y orientaciones en las que el patrón fue fotografiado.
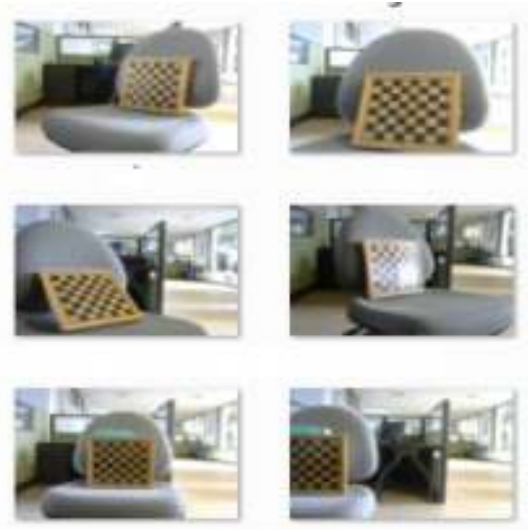

Fig 3. Imagen del Patrón Geométrico para calibración de la cámara desde diferentes perspectivas

En total se tomaron 61 fotografías del patrón, desde una distancia fija, asemejando la posición que la cámara tendría dentro del estanque de las tilapias. Utilizando la herramienta 'Camera calibration toolbox for Matlab R2015a (8.5.0.197613)' se encontraron los parámetros extrínsecos e intrínsecos de la cámara, en función

de las 61 fotografías del patrón fotográfico.

En la figura 4 se muestra el toolbox para encontrar los parámetros de la cámara y a continuación la figura 5 , donde el toolbox realiza el análisis del patrón geométrico representado en la fotografía.

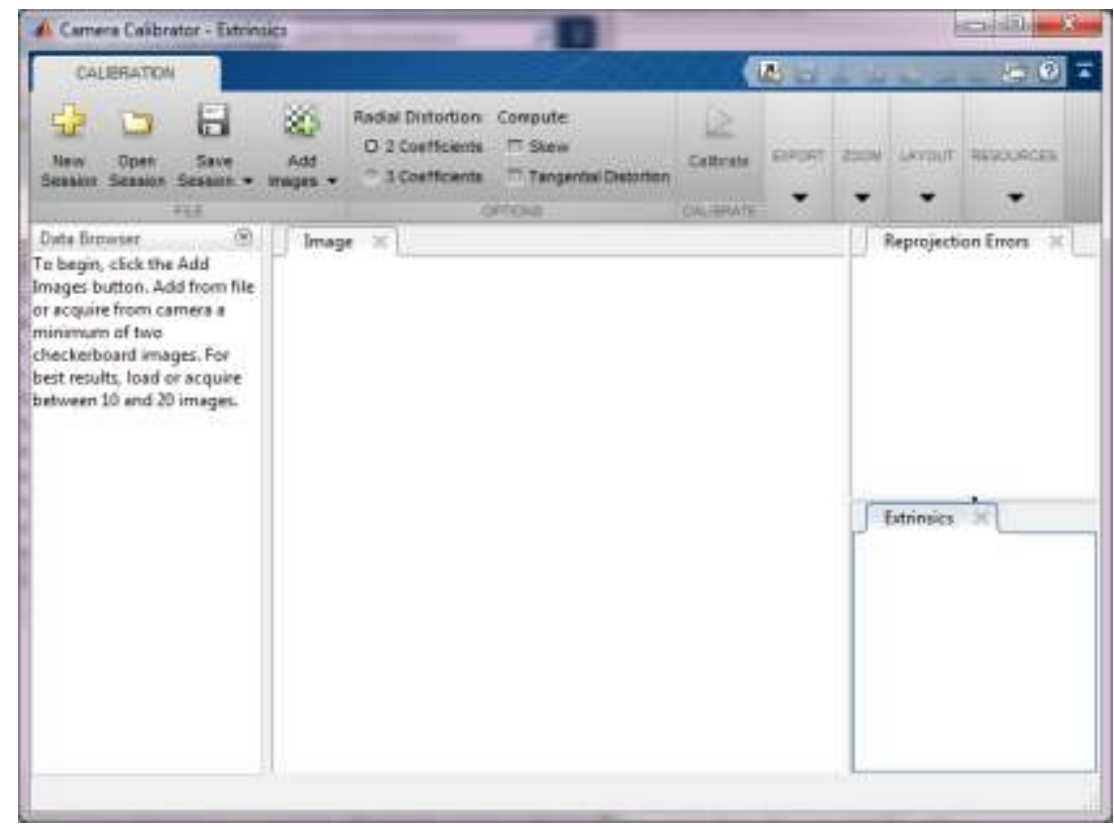

Fig 4. Imagen Camera calibration toolbox for Matlab 


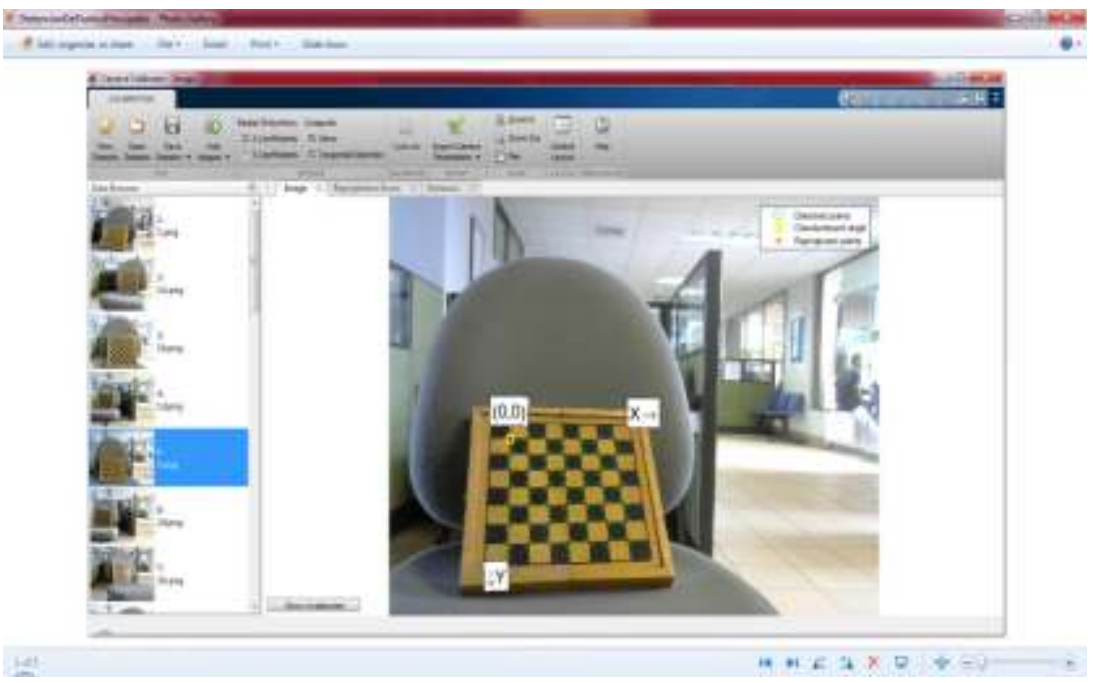

Fig 5. Imagen de identificación de coordenadas geométricas

Con la figura 6 se observa que se ha podido identificar automáticamente el patrón geométrico de la fotografía y la orientación espacial con respecto al entorno, es decir, la dirección $\mathrm{x}$ y la dirección y.

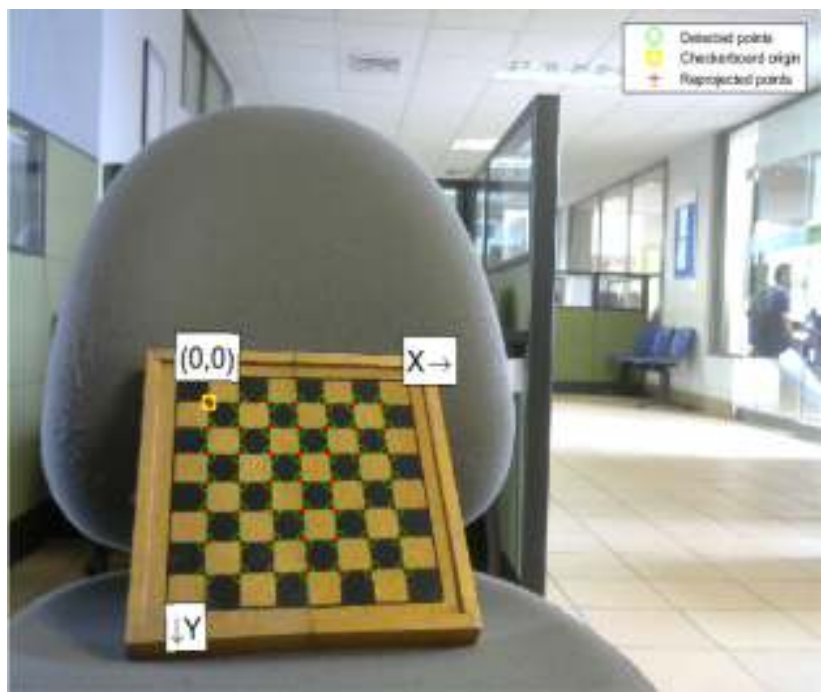

Fig 6. Imagen del cálculo de orientación espacial de la cámara con entorno

Con la anterior información el toolbox puede calcular la orientación espacial de la cámara con respecto a todas las fotografías tomadas, como se presenta en la figura 7.

El toolbox también entregó la información del error medio de la posición real del patrón con la posición en el sensor óptico de la cámara -figura 8-, para conocer que incertidumbre tendrán los cálculos biométricos obtenidos de las fotografías de las tilapias.

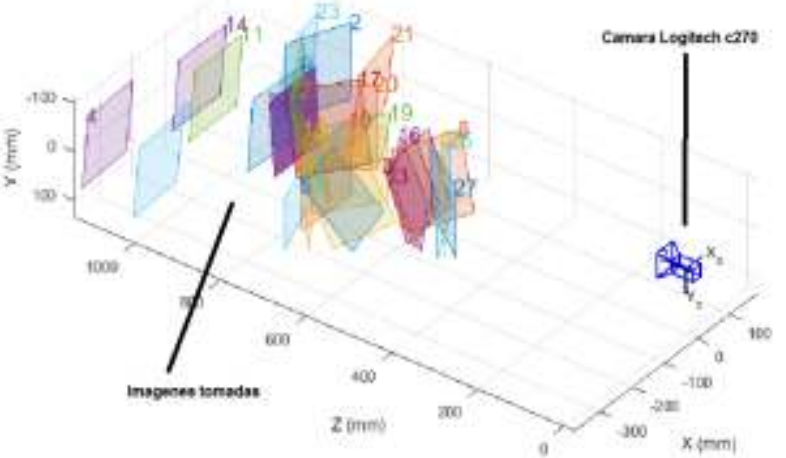

Fig 7. Gráfica del cálculo del error medio de la posición del patrón

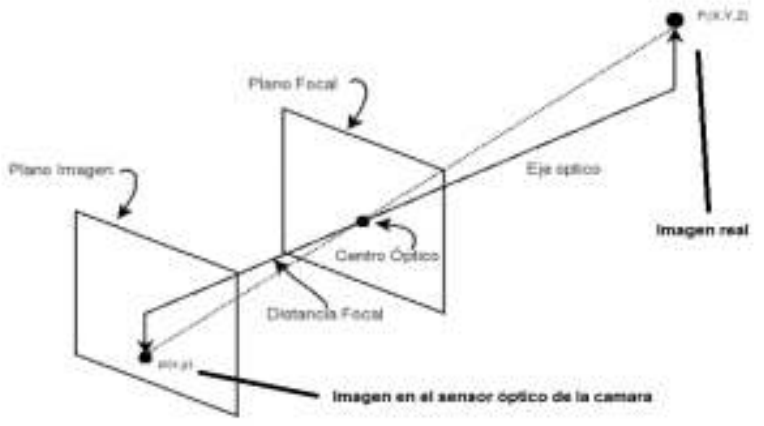

Fig 8. Gráfica del Modelo Geométrico del sistema óptico de la cámara 
La dispersión del error de la posición real del patrón con respecto a la posición en el sensor óptico de la fotografía tomada por la cámara se encuentra entre un rango de -2 a 2 pixeles, como se puede ver en la gráfica de la figura 9 .

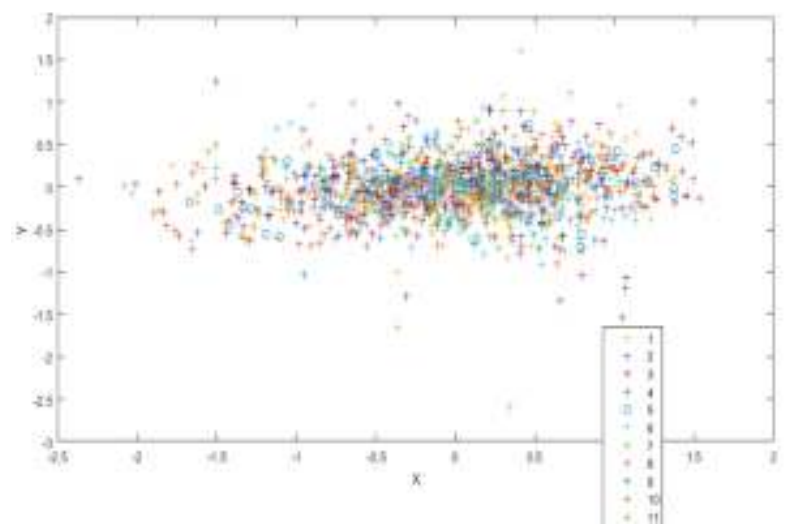

Fig 9. Grafica Dispersión del error de la posición real del patrón

\subsection{Parámetros Extrínsecos}

Matriz de rotación:
Con la anterior información el toolbox entrega los siguientes valores de los parámetros extrínsecos e intrínsecos de la cámara Logitich C270.

\subsection{Parámetros Intrínsecos} Longitud focal en pixeles:

[1393.6500+/-5.5381 1418.8207+/-5.4016]

Punto Principal en el sensor óptico en pixeles:

[658.0915+/-7.8653 511.3129+/-8.2107]

Inclinación sensor óptico en pixeles:

[-2.0173+/-1.0781]

Distorsión radial en pixeles:

$[0.0401+/-0.0166 \quad-0.3673+/-0.1035]$

Distorsión tangencial en pixeles:

$[0.0085+/-0.0022 \quad 0.0052+/-0.0021]$

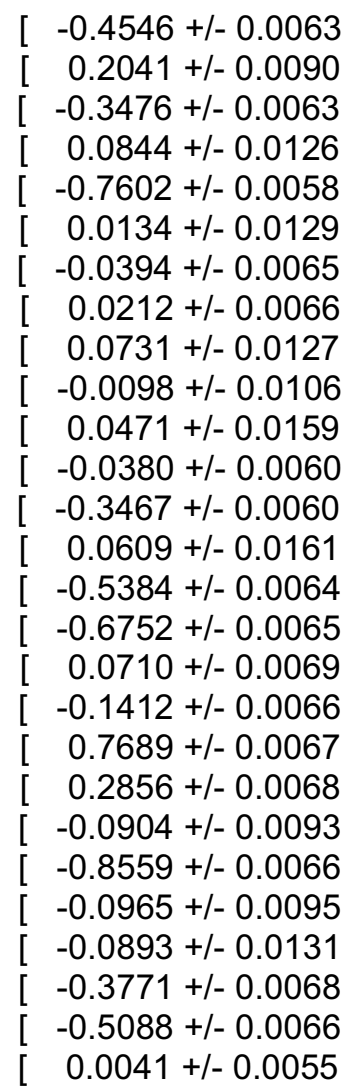

$0.7788+/-0.0063$

$0.2846+/-0.0088$

$-0.0649+/-0.0063$

$-0.0638+/-0.0118$

$-0.2369+/-0.0055$

$-0.1256+/-0.0143$

$-0.5372+/-0.0064$

$0.6093+/-0.0062$

$-0.0385+/-0.0125$

$0.0308+/-0.0104$

$-0.0841+/-0.0157$

$0.5930+/-0.0059$

$-0.3211+/-0.0060$

$-0.0842+/-0.0159$

$-0.6216+/-0.0058$

$-0.1475+/-0.0059$

$0.7709+/-0.0064$

$-0.8917+/-0.0061$

$0.1331+/-0.0059$

$0.9843+/-0.0061$

$-0.2473+/-0.0081$

$-0.2427+/-0.0059$

$-0.2487+/-0.0088$

$-0.0472+/-0.0131$

$-0.3659+/-0.0063$

$-0.4999+/-0.0061$

$-0.9588+/-0.0057$

$1.6033+/-0.0030]$
$1.6229+/-0.0012]$
$-0.0173+/-0.0009]$
$0.0169+-0.0018]$
$-0.0742+/-0.0019]$
$1.5872+/-0.0016]$
$-0.0621+/-0.0016]$
$1.6273+/-0.0019]$
$1.5856+/-0.0009]$
$1.5691+/-0.0010]$
$1.5836+/-0.0010]$
$0.0311+/-0.0021]$
$-0.0817+/-0.0011]$
$1.5798+/-0.0010]$
$1.4635+/-0.0019]$
$1.3761+/-0.0017]$
$1.6617+/-0.0024]$
$1.5829+/-0.0022]$
$1.3531+/-0.0026]$
$1.6837+/-0.0031]$
$1.5779+/-0.0014]$
$1.2916+/-0.0024]$
$1.5808+/-0.0013]$
$0.0258+/-0.0008]$
$1.5120+/-0.0013]$
$1.4698+/-0.0018]$
$-0.0682+/-0.0027]$


Matriz de traslación $(\mathrm{mm})$ :

$$
\begin{aligned}
& \text { [ } \quad-4.5791+/-4.2573 \\
& \text { [ } 142.5041+/-5.0430 \\
& \text { [ }-86.9342+/-3.5959 \\
& \text { [ }-364.6714+/-6.3911 \\
& \text { [ }-185.4032+/-4.2743 \\
& \text { [ }-225.2568+/-5.7787 \\
& \text { [ }-82.0980+/-4.8242 \\
& \text { [ } 100.8047+/-3.3983 \\
& \text { [ } 43.2925+/-5.2260 \\
& \text { [ }-11.5695+/-4.3716 \\
& \text { [ } \quad 43.6673+/-6.0215 \\
& {[-103.5318+/-4.4730} \\
& {[-108.0869+/-3.4786} \\
& \text { [ } 48.7128+/-6.2487 \\
& \text { [ }-93.7307+/-4.6125 \\
& 6.0104+/-3.7745 \\
& \text { [ } 122.1101+/-4.8118 \\
& \text { [ }-78.2538+/-4.4683 \\
& \text { [ } \quad 68.6585+/-4.1644 \\
& \text { [ } 130.0631+/-4.6370 \\
& \text { [ } 187.0679+/-4.6871 \\
& 2.1151+/-5.2212 \\
& \text { [ } 137.1804+/-5.4400 \\
& \text { [ }-79.6338+/-5.1347 \\
& \text { [ } 46.1700+/-3.2875 \\
& \text { [ } 38.0004+/-3.3472 \\
& \text { [ }-66.2774+/-2.7511
\end{aligned}
$$

$28.3212+/-4.3535$

$102.1211+/-5.1560$

$-12.6148+/-3.7051$

$-73.5066+/-6.6925$

$22.6845+/-4.4691$

$-62.0517+/-5.8698$

$-100.2450+/-4.9495$

$-9.5895+/-3.4903$

$-47.7333+/-5.3587$

$-19.2482+/-4.4790$

$-58.4587+/-6.1762$

$-18.6587+/-4.6225$

$-12.9021+/-3.5882$

$-61.2252+/-6.4095$

$-24.4074+/-4.7440$

$-17.7679+/-3.8785$

$-8.8784+/-4.9433$

$-9.5829+/-4.5968$

$-9.0430+/-4.2897$

$-6.9841+/-4.7647$

$-78.6013+/-4.8186$

$-16.0758+/-5.3621$

$-99.2903+/-5.5762$

$-40.6602+/-5.2782$

$-21.1974+/-3.3810$

$-22.0370+/-3.4408$

$-14.6118+/-2.8371$

$755.0252+/-3.0374$ ]
$886.8206+/-4.0976$ ]
$638.3497+/-2.4468$ ]
$1117.4757+/-5.6192$ ]
$756.7601+/-3.0397$ ]
$1000.9159+/-4.9002$ ]
$852.9810+/-3.6331]$
$600.7494+/-2.5899]$
$925.7595+/-4.1523]$
$773.8584+/-3.2398]$
$1066.9255+/-4.8812]$
$794.7355+/-2.9235]$
$615.5725+/-2.4264]$
$1107.0982+/-5.0954]$
$816.7916+/-3.1612]$
$671.4943+/-2.4989]$
$851.0620+/-3.6690]$
$793.1339+/-3.3679]$
$739.0592+/-3.4293]$
$819.0937+/-3.6198]$
$823.4545+/-3.9113]$
$926.6654+/-3.4579]$
$960.6095+/-4.2545$ ]
$910.3072+/-3.7572]$
$585.0372+/-2.3089]$
$595.6151+/-2.2811]$
$487.1227+/-2.1188]$

$755.0252+/-3.0374]$

$638.3497+/-2.4468$

$1117.4757+/-5.6192$

$756.7601+/-3.0397$

$1000.9159+/-4.9002$

$852.9810+/-3.6331$

$600.7494+/-2.5899$ ]

$925.7595+/-4.1523$ ]

$773.8584+/-3.2398$

$1066.9255+/-4.8812]$

$794.7355+/-2.9235$

$615.5725+/-2.4264$

$107.0982+/-5.0954$

$816.7916+/-3.1612$

$671.4943+/-2.4989$

$851.0620+/-3.6690$

$793.1339+/-3.3679$

$819.0937+/-3.6198$

$823.4545+/-3.9113$ ]

$926.6654+/-3.4579$

$960.6095+/-4.2545]$

$910.3072+/-3.7572$

$595.6151+/-2.2811$

$487.1227+/-2.1188$ ]
Con los parámetros encontrados de la cámara Logitech c270 se buscará el modelo matemático que relacione las medidas reales biométricas de las tilapias con el análisis de las imágenes digitalizadas. El modelo matemático tendrá en cuenta el Modelo Pinhole aplicado a las cámaras, tal y como lo muestra el esquema de la figura 10.

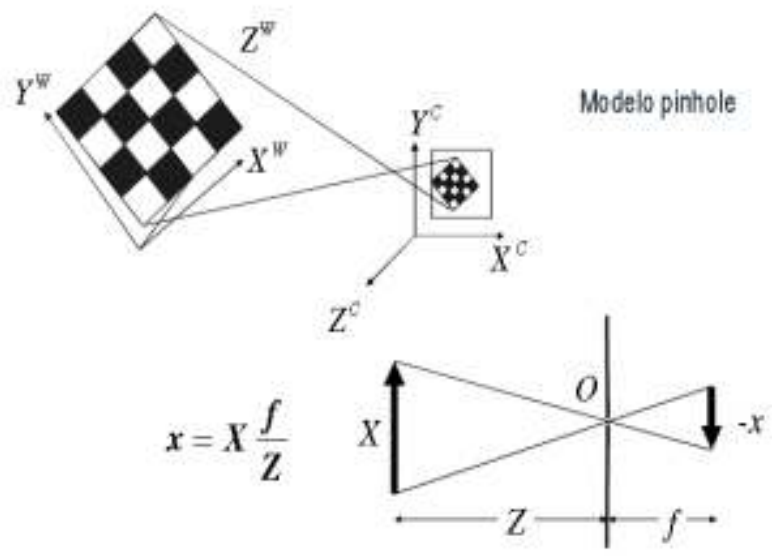

Fig 10. Representación del modelo Pinhole de la cámara

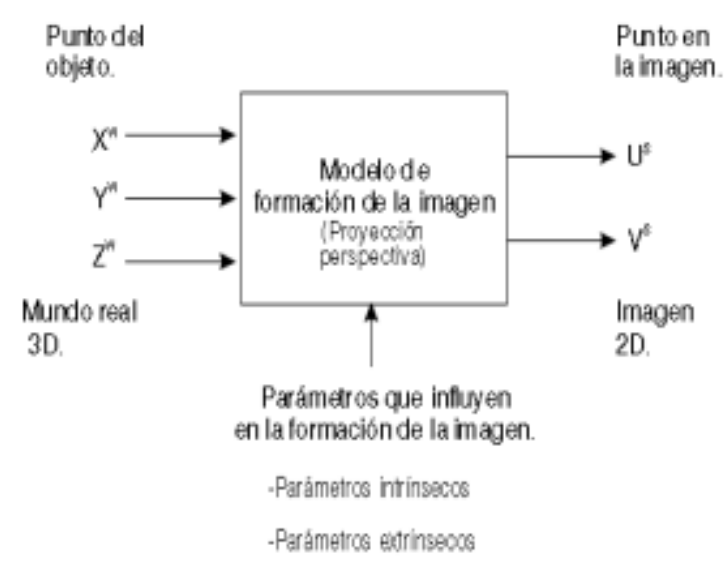

Fig 11. Gráfica de entradas y salidas del modelo matemático de la cámara

Con la información de los parámetros intrínsecos, extrínsecos se construirá el modelo matemático que tendrá la siguiente forma: cualquier punto tridimensional $(X W, Y W, Z W)$ de las tilapias puede llevarse a un sistema de coordenadas de la 
cámara Logitech c270 (XC, YC, ZC), mediante una matriz de rotación $R$ y otra de translación $T$ : como se representa en la siguiente figura 12 .

$$
\left(\begin{array}{c}
X_{C} \\
Y_{C} \\
Z_{C}
\end{array}\right)=R\left(\begin{array}{c}
X_{W} \\
Y_{W} \\
Z_{W}
\end{array}\right)+T=\left(\begin{array}{lll}
r_{1} & r_{2} & r_{3} \\
r_{4} & r_{5} & r_{6} \\
r_{7} & r_{8} & r_{9}
\end{array}\right)\left(\begin{array}{c}
X_{W} \\
Y_{W} \\
Z_{W}
\end{array}\right)+\left(\begin{array}{c}
T_{x} \\
T_{Y} \\
T_{z}
\end{array}\right)
$$

Fig 12. Grafica de la ecuación matricial del modelo matemático de la cámara

Los valores de $\mathrm{R}$ y $\mathrm{T}$ son los parámetros extrínsecos de la cámara Logitech C270.

Luego, este sistema coordenado en el espacio tridimensional, se debe llevar al espacio bidimensional de las imágenes en el sensor óptico de la cámara, sin tener en cuenta las distorsiones radial y tangencial, para hallar las coordenadas de la imagen $(x b, y b)$.

$$
\left(\begin{array}{c}
x_{b} \\
y_{b} \\
1
\end{array}\right)=k\left(\begin{array}{ccc}
f_{a} & s & C_{x} \\
0 & f_{c y} & C_{y} \\
0 & 0 & 1
\end{array}\right)\left(\begin{array}{l}
X_{c} \\
Y_{c} \\
Z_{c}
\end{array}\right)
$$

Fig 13. Otra Representación del modelo matemático de la cámara

Siendo fcx y fcy los valores de la distancia focal de la cámara Logitech C270 medidas en pixeles, Cx y Cy es el punto central del sensor óptico y $S$ es el valor del área del sensor óptico. Con lo anterior se obtendrán los valores biométricos en unidades de centímetros, de las imágenes tomadas de las tilapias.

\subsection{Calibración de los Parámetros} Extrínsecos de la Cámara Logitech c270 Utilizando la Herramienta "Camera Calibration Tolbox for Matlab"

En la gráfica de la figura 14 se muestran los pasos realizados para obtener los parámetros extrínsecos a la cámara con respecto a la posición donde será ubicada en el estanque. Teniendo en cuenta que el estanque tiene un ancho de $90 \mathrm{~cm}$, el enfoque de la cámara se colocó a una distancia de $45 \mathrm{~cm}$ con respecto al sensor óptico de la misma. Se tomaron fotos de diferentes longitudes y a una distancia de alrededor de $45 \mathrm{~cm}$ para encontrar la proporción entre la longitud real del objeto con la longitud captada en pixeles por el sensor óptico de la cámara. Estos valores se llevaron a un libro de Excel donde se encontró la función matemática que relaciona longitudes reales con valores de pixeles en el sensor óptico. Para obtener la función matemática se tuvieron en cuenta los valores entregados por las calibraciones intrínsecas realizadas en el apartado anterior.

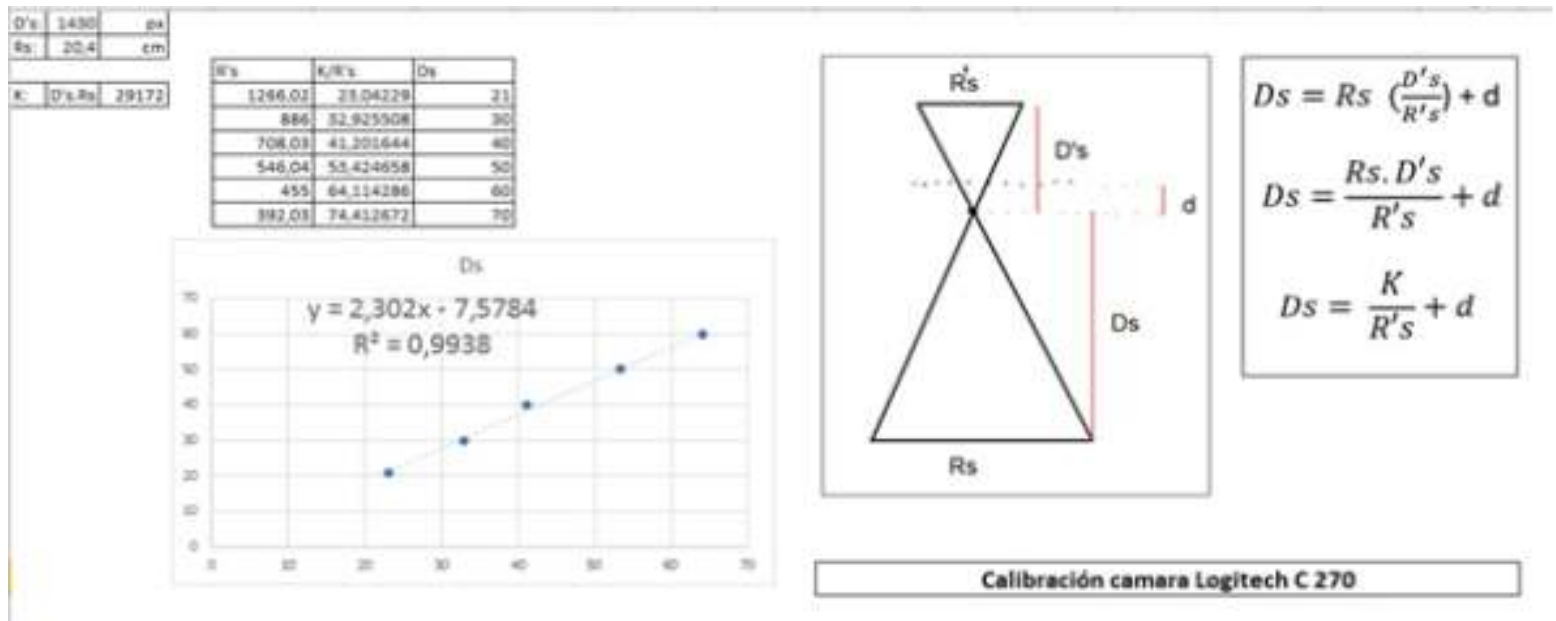

Fig 14. Gráfica de calibración extrínseca con relación a la posición de la cámara en el estanque

\subsection{Desarrollar un Algoritmo para} Determinar Medidas Biométricas en Peces.

Con base en datos entregados por los muestreos poblacionales y fotos tomadas en el estanque a las tilapias, como lo muestran las figuras 15 a 17 , se obtuvo una tabla que relaciona la longitud del pez con su peso. Estos valores se llevaron a un libro de Excel donde se encontró la función matemática. Para estas medidas se utilizó un ictiometro con una precisión de $0,1 \mathrm{~cm}$ y una gramera digital con una precisión de 0,01 g. 


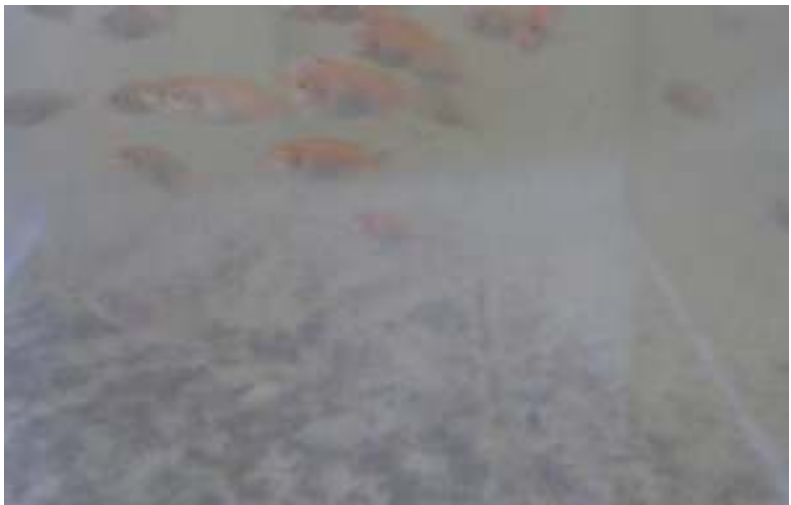

Fig 15. Muestreo poblacional.

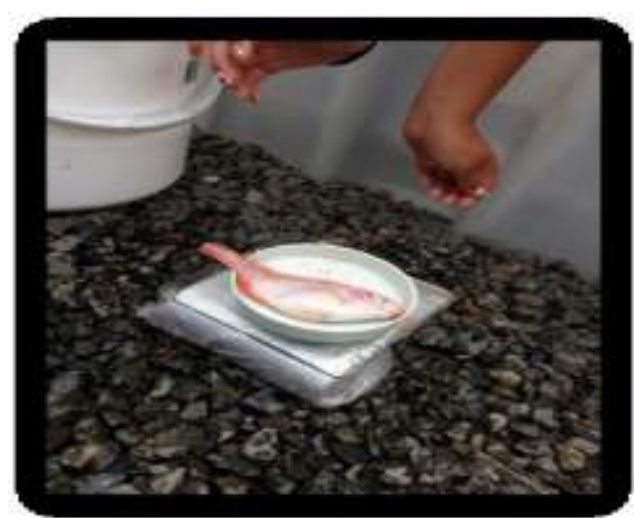

Fig 16. Imagen de obtención de parámetros biométricos.
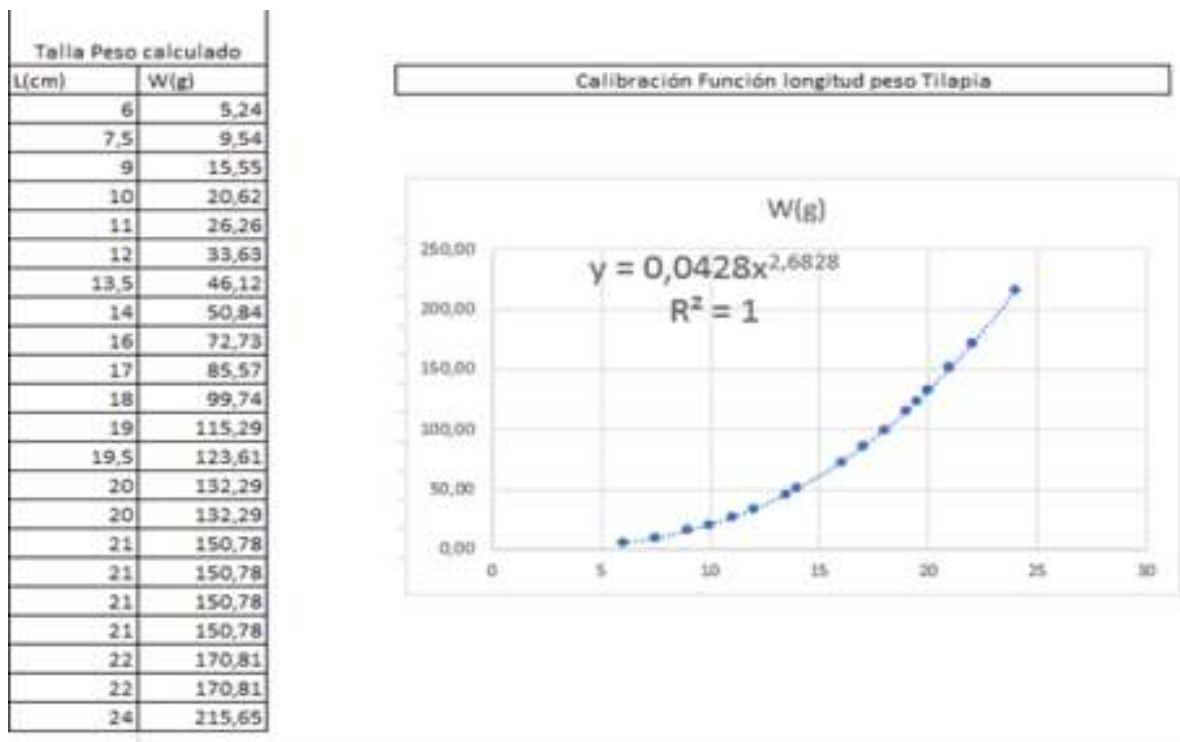

Fig 17. Gráfica de obtención de la ecuación relación longitud vs peso de la tilapia

\section{CONCLUSIONES}

Se obtuvieron los parámetros intrínsecos y extrínsecos de la cámara Logitech C270 aplicando la técnica flexible de Zhang y por medio de la herramienta 'Camera calibration toolbox de Matlab, los cuales se utilizaron para desarrollar el modelo matemático según las relaciones matriciales (1) y (2), cuya ecuación es la siguiente:

$$
\text { Los } y=0,0428 x^{2,6828} \text { porcentajes de }
$$
error para el cálculo de los parámetros intrínsecos y extrínsecos no son significativos y no tendrán un efecto en el modelo matemático, como se observa en los siguientes valores: [ $\mathrm{X}$ : $-0.4546+/-0.0063$ Y: $0.7788+/-0.0063 \quad Z: 1.6033+/-0.0030$ ]

\section{REFERENCIAS}

Cyganek, B., \& Siebert, J. P. (2011). An introduction to 3D computer vision techniques and algorithms. John Wiley \& Sons.

Gardel, V. A. (2004). Calibración de cámaras con objetivos de distancia focal fija.

Torres, F., Gil, P., Puente, S. T., \& Aracil, R. (2002). Robots y sistemas sensoriales. Pearson Educación.

Tsai, R. (1987). A versatile camera calibration technique for highaccuracy $3 \mathrm{D}$ machine vision metrology using off-the-shelf TV cameras and lenses. IEEE Journal on Robotics and Automation, 3(4), 323-344.

Vega Perez, J. M. (2008). Reconstrucción de escenas en 3D.

Zhang, Z. (2000). A flexible new technique for camera calibration. IEEE Transactions on pattern analysis and machine intelligence, 22(11), 1330-1334. 